Chirurg 2012 $\cdot 83: 821$

DOI 10.1007/s00104-012-2359-x

Online publiziert: 23 . August 2012

(c) Springer-Verlag 2012

O. Strobel · M.W. Büchler

Klinik für Allgemein-, Viszeral- und Transplantationschirurgie, Universität Heidelberg

\title{
Mesh-verstärktes Stapeln bei der Pankreaslinksresektion
}

\section{Lösung des Problems Pankreasfistel?}

\section{Originalpublikation}

Hamilton NA, Porembka MR, Johnston FM et al (2012) Mesh reinforcement of pancreatic transection decreases incidence of pancreatic occlusion failure for left pancreatectomy: a single-blinded, randomized controlled trial. Ann Surg 255:1037-1042

\section{Hintergrund und Fragestellung}

Die Morbidität nach Pankreaslinksresektion wird maßgeblich durch die Häufigkeit der postoperativen Pankreasfistel (POPF) bestimmt, die bei etwa 30\% der Patienten auftritt und ein bisher ungelöstes Problem in der Pankreaschirurgie darstellt.

In einer randomisiert-kontrollierten Studie verglichen Hamilton et al. die Ergebnisse nach Durchtrennung/Verschluss des Pankreas mittels Stapler mit und ohne Mesh-Verstärkung.

\section{Methoden}

Hundert Patienten wurden intraoperativ in die Gruppen „Mesh-Verstärkung“ (Mesh; n=54) und „keine Mesh-Verstärkung“ (ohne Mesh; n=46) randomisiert. Alle Patienten wurden am selben Zentrum operiert, davon $96 \%$ von 3 Chirurgen. Sieben Patienten (6,5\%) wurden ausgeschlossen wegen Bedenken des Operateurs bezüglich der Verwendung eines Staplers zur Durchtrennung des Pankreas. Der primäre Endpunkt war das Auftreten einer klinisch relevanten POPF (Grad B und C, ISGPF [International Study Group on Pancreatic Fistula]-Klassifikation). Sekundäre Endpunkte waren das Auftreten aller POPF, die Dauer bis zum Drainagezug, die Notwendigkeit weiterer Eingriffe und andere Komplikationen.

\section{Ergebnisse}

Die beiden Gruppen waren bezüglich demographischer und intraoperativer Parameter sowie bezüglich der Indikationen prinzipiell vergleichbar. Bei den Komorbiditäten zeigte sich allerdings ein signifikant höherer Body-Mass-Index in der Gruppe „ohne Mesh“. Die Autoren berichten eine signifikant verminderte Rate an klinisch relevanten POPF in der Mesh-Gruppe (1,9\% mit Mesh vs. $20 \%$ ohne Mesh). Die Häufigkeit aller POPF (inklusive Grad A) war nicht signifikant unterschiedlich (39\% mit Mesh vs. 57\% ohne Mesh). In der Gruppe ohne Mesh war signifikant häufiger eine Wiedereinlage einer Drainage notwendig (1,9\% mit Mesh vs. 20\% ohne Mesh). In der Gruppe ohne Mesh war die Zeit bis zur definitiven Entfernung der Drainage signifikant verlängert (9 Tage mit Mesh vs. 17 Tage ohne Mesh). Intraoperative Komplikationen waren in der Mesh-Gruppe häufiger ( $11 \%$ vs. $4,3 \%)$. Anderer postoperative Komplikationen als die POPF waren in der Gruppe ohne Mesh tendenziell häufiger (39\% mit Mesh vs. $61 \%$ ohne Mesh).

\section{Schlussfolgerung}

In der vorliegenden Studie führt das Mesh-verstärkte Stapeln zu einer erstaunlich deutlichen Reduktion klinisch relevanter POPF. Bei der Interpretation dieser Ergebnisse sind folgende Punkte wichtig: - In der Klassifikation der POPF führt eine interventionelle Drainageneinlage automatisch zu einem Upgrade einer „harmlosen“ Typ-A-Fistel zu einer „klinisch relevanten“ Typ-B-Fistel. Die Frequenz der klinisch relevanten POPF ist daher ganz entscheidend vom Vorgehen hinsichtlich initialem Zug und späterem Wiedereinlegen einer Drainage abhängig. Die Autoren machen hierzu keine Angaben. Da sich die Zahl der klinisch relevanten Fisteln mit der Zahl wiedereingelegter Drainagen deckt, könnten die Ergebnisse durch ein nicht standardisiertes Vorgehen verfälscht sein.

- Es handelt sich um eine relativ kleine, unizentrische Studie. Fast alle Patienten wurden von nur 3 Chirurgen operiert. Die Ergebnisse in der Gruppe ohne Mesh mit 57\% POPF sind schlechter als in aktuellen multizentrischen Studien.

Die deutliche Reduktion der Fistelrate in der Mesh-Gruppe muss in weiteren multizentrischen Studien mit geeignetem Design und genauer Angabe der Kriterien hinsichtlich Drainagezug und -wiedereinlage bestätigt werden. Zusätzlich ist ein Vergleich mit anderen Techniken sinnvoll, wie der serosalen Deckung des Pankreasabsetzungsrandes.

Die vorliegende Studie weist auf ein Potential des Mesh-verstärkten Stapelns zur Reduktion relevanter POPF hin, reicht aber für eine Empfehlung dieser Technik noch nicht aus.

\section{Korrespondenzadresse}

PD Dr. O. Strobel

Klinik für Allgemein-, Viszeral- und Transplantationschirurgie, Universität Heidelberg, Im Neuenheimer Feld 110, 69120 Heidelberg Oliver.Strobel@med.uni-heidelberg.de

Interessenkonflikt. Der korrespondierende Autor gibt für sich und seinen Koautor an, dass kein Interessenkonflikt besteht. 